\begin{tabular}{|c|c|}
\hline 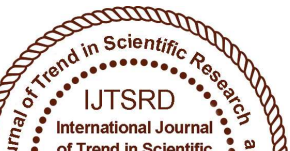 & $\begin{array}{l}\text { International Journal of Trend in Scientific } \\
\text { Research and Development (IJTSRD) }\end{array}$ \\
\hline 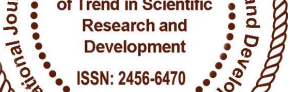 & International Open Access Journal \\
\hline 000 & ISSN No: 2456 - 6470 | www.ijtsrd.com | Volume - 2 | Issue - 3 \\
\hline
\end{tabular}

\title{
Review:Wavelet transform based electroencephalogram methods
}

\author{
Miss. N. R. Patil \\ PG Student, Department of Electronics Engineering \\ in PVPIT, Budhgaon, Sangli, Maharashtra, India
}

\author{
Prof. S. N. Patil \\ Project Guide, Department of Electronics Engineering \\ in PVPIT, Budhgaon, Sangli, Maharashtra, India
}

\begin{abstract}
In this paper, EEG signals are the signatures of neural activities. There have been many algorithms developed so far for processing EEG signals.

The analysis of brain waves plays an important role in diagnosis of different brain disorders. Brain is made up of billions of brain cells called neurons, which use electricity to communicate with each other. The combination of millions of neurons sending signals at once produces an enormous amount of electrical activity in the brain, which can be detected using sensitive medical equipment such as an EEG which measures electrical levels over areas of the scalp. The electroencephalogram (EEG) recording is a useful tool for studying the functional state of the brain and for diagnosing certain disorders. The combination of electrical activity of the brain is commonly called a Brainwave pattern because of its wave-like nature.

EEG signals are low voltage signals that are contaminated by various types of noises that are also called as artifacts. Statistical method for removing artifacts from EEG recordings through wavelet transform without considering SNR calculation is proposed.
\end{abstract}

Keywords: EEG, Discreet Wavelet Transform, EOG, SNR, MSE

\section{INTRODUCTION}

EEG recordings therefore, complete knowledge about overall activity of the millions of neurons in the brain. Brain is one of the most important organs of humans, for controlling the coordination of human muscles and nerves. The EEG is the recording of brain's electrical activity. EEG is one commonly used non-invasive facility to investigate the intricacy of human brain.
The EEG is used in the evaluation of brain disorders. It is also used to evaluate people who are having problems associated with brain. An EEG is also used to determine brain death. [1]

Electro-encephalogram (EEG) is the electrical activity of brain cell groups in the cerebral cortex or the scalp surface. The mechanism of EEG is a complex random signal within the brain activities, it is in the cerebral cortex of the synthesis of millions of nerve cells. Brain electrical activity is generated by electric volume conductor (the cortex, skull, meninges, and scalp). It reflects the electrical activity of brain tissue and brain function. Different state of mind and the cause of the cerebral cortex in different locations reflect the different EEG.[1]

EEG signals are having very small amplitudes and because of that they can be easily contaminated by noise . The noise can be electrode noise or can be generated from the body itself. The noises in the EEG signals are called the artifacts and these artifacts are needed to be removed from the original signal for the proper analysis of the EEG signals .The various types of noises that can occur in the signals during recordings are the electrode noise, baseline movement, EMG disturbance and so on .We need to remove these noises from the original EEG signal for proper processing and analysis of the diseases related to brain. [2]

Despite the impressive advancements in brain imaging, interpreting brain waves remains remains difficult: brain imaging data are often complex and vast; it is often impossible to visually inspect all data. 
Therefore, techniques from signal processing may play an increasingly important role in the area of brain imaging.[3]

\section{LITERATURE REVIEW}

\section{Denoising EEG Signal Using Wavelet Transform}

R. Princy, P. Thamarai, B.Karthik, given various ideas for Electroencephalogram (EEG) signal is the recording of spontaneous electrical activity of the brain over a small interval of time. Signals are produced by bombardment of neurons within the brains which are measured and evaluated by EEG. EEG signals are low voltage signals that are contaminated by various types of noises that are also called as artifacts. As these signals are used to diagnose various types of brain related diseases like narcolepsy, Sleep apneasyndrome, Insomnia and parasomnia it becomes necessary to make these signals free from noise for proper analysis and detection of the diseases.. [1]

\section{Wavelet Time-frequency Analysis of Electro- encephalogram (EEG) Processing}

Zhang xizheng, Yin ling, Wang weixiong, In this paper, the basic idea is to use the characteristics of multi-scale multi-resolution, using four different thresholds to wipe off interference and noise after decomposition of the EEG signals. By analyzing the results, understanding the effects of four different methods, it comes to a conclusion that the wavelet denoising and soft threshold is a better conclusion. [2]

\section{Topics in Brain Signal Processing}

Justin Dauwels_ and Franc, ois Vialatte, this paper provides an introduction to the area of brain signal processing, and also serves as an introductory presentation for the special session entitled Advanced Signal Processing of Brain Signals: Methods and Applications at APSIPA 2010. Several topics related to the processing of brain signals are discussed: preprocessing, inverse modeling (a.k.a. source modeling), and signal decoding. The papers in the special session are centered around those three topics.[3]

\section{EEG Purging Using Labview Based Wavelet Analysis}

Jigar D. Shah, M. S. Panse, this paper provides the removal of artifact from scalp EEGs is of considerable importance for analysis of underlying brainwave activity. The presence of artifacts such as muscle activity, eye blinks, pulse signals and line noise in electroencephalographic (EEG) recordings obscures the underlying processes. These artifacts sources increase the difficulty in analyzing the EEG. For this reason, it is necessary to design a procedure to decrease such artifacts in EEG.[4]

\section{Subtraction using linear regression}

When dealing with predictable noise that can be recorded independently on a separate channel, it is possible to remove the noise from the data by estimating the amount of noise transtered to data using linear regression and then subtracting it. A typical example is the noise produced by blinks and eye movements. To remove such noise, linear regression is computed between each data channel and nuisance channels used to record horizontal (HEOG - difference between voltages recorded above and below eyes), vertical (VEOG - difference between voltages recorded at the left and right outer canthi of the eyes) and radial (REOG - difference between average voltage at the eyes and EEG reference) movement. The estimated $\beta$ coefficients are then used to subtract values from each nuisance channel multiplied with corresponding $\beta$ coefficient from the measured data channel.[5]

\section{Subtraction using adaptive filtering}

As EOG signals are mostly of lower frequency than the cerebellar signal of interest, the problem of EOG signals contamination by signal of interest can be somewhat reduced by low-pass filtering the EOG channels before applying substraction using linear regression, problem of removing cerebellar signal of interest along with EOG in subtraction using linear regression.[6]

\section{Subtraction using data decomposition}

The only way to fully remove the nuisance signal while avoiding removal of signal of interest is to efficiently estimate and remove only the nuisance signal related to a specific source of noise. A number of methods for estimating specific sources of EEG signal developed and tested in the recent years fall under the umbrella of blind source separation (BSS). The key assumption of BBS is that the observed signal can be understood as a mixture of original source signals. The specific methods differ in the algorithms and information used to estimate the mixing matrix and the original source signals.

Second order statistics (SOS) methods are based on the assumption that the original source signals are 
uncorrelated and aim to decompose the observed signal into a number of uncorrelated components. Probably the most widely known method is pricipal component analysis (PCA), which decomposes the time series into a number of orthogonal (uncorrelated) sources with decreasing significance, such that a small number of components contain most of the variance of the measured signal. PCA is most often used as a data reduction method.[7]

Recently, new SOS methods have been developed that make use of the temporal structure of the signal by relying on time-lagged covariance matrices. Two representatives of this approach are algorithm for multiple unknown signals extraction (AMUSE) [8] and second-order blind identification (SOBI).[9]

\section{EEG Analysis - Automatic Spike Detection}

In the diagnosis and treatment of epilepsy, an electroencephalography (EEG) is one of the main tools. However visual inspection of EEG is very time consuming. Automatic extraction of important EEG features saves not only a lot of time for neurologist, but also enables a whole new level for EEG analysis, by using data mining methods. In this work we present and analyse methods to extract some of these features of EEG - drowsiness score and centrotemporal spikes.[10]

DEAP: A Database for Emotion Analysis Using Physiological Signals

Sander Koelstra, Mohammad Soleymani, Ashkan Yazdani, Anton Nijholt, this paper provides the electroencephalogram (EEG) and peripheral physiological signals of 32 participants were recorded as each watched 40 one-minute long excerpts of music videos. Participants rated each video in terms of the levels of arousal, valence, like/dislike, dominance, and familiarity. For 22 of the 32 participants, frontal face video was also recorded. A novel method for stimuli selection is proposed using retrieval by affective tags from the last.fm website, video highlight detection, and an online assessment tool. An extensive analysis of the participants' ratings during the experiment is presented. Correlates between the EEG signal frequencies and the participants' ratings are investigated. Methods and results are presented for single-trial classification of arousal, valence, and like/dislike ratings using the modalities of EEG, peripheral physiological signals, and multimedia content analysis. Finally, decision fusion of the classification results from different modalities is performed.[11]

\section{Multispectrum Approach in Quantitative EEG: Accuracy and Physical Effort}

A. Lay-Ekuakille, P. Vergallo, D. Caratelli, F. Conversano, S. Casciaro, and A. Trabacca, this paper provides the detection of neurophysiological features by means of electroencephalogram (EEG) is one of the most recurrent medical exams to be performed on human beings. As it is noticed, EEG trials are not always sufficient to deliver a clear and precise diagnosis for many pathologies. Hence it must be integrated with other exams. But we can use all additional instrumental exams to improve the quality of the diagnosis since there are other constraints, namely, financial, medical and individual. This work presents an original implementation of EEG signal processing using filter diagonalization method (FDM) to build a bispectrum and contour representation in order to discover possible abnormalities hidden in the signal for aided-diagnosis.[12]

\section{EEG De-noising using SURE Thresholding based on Wavelet Transforms}

G. Geetha, Dr.S.N.Geethalakshmi, this paper provides the Electroencephalogram (EEG) is a biological signal that represents the electrical activity of the brain. However, the presence of artifacts like electrooculogram (EOG), Electrocardiogram (ECG), electromyogram (EMG) and powerline noise in the EEG signal is a major problem in the study of brain potentials. Hence, these superfluous signals are needed to be removed. There are various methods for removal of artifacts. [13]

Analysis of Finger Movements Using EEG Signal

C.Vigneshwari, V.Vimala, S.Vaira Vignesh, G.Sumithra,in this paper provided that the electroencephalogram (EEG) signals of left and right hand finger movements, an application of BrainComputer Interface (BCI). Discrete Wavelet Transform is used for Feature extraction, which separates Alpha and Beta band of frequencies of EEG Signal. Various noises pose a major problem in recognizing the EEG signal. Random noise is generally difficult to be removed using typical filtering methods. Ten features which include both time domain and frequency domain are evaluated in a noisy environment, for various Signal-to-Noise ratios. [14] 


\section{CONCLUSION}

In this paper, time-frequency analysis toolbox function tfrsp is used in analysis spectrogram of EEG. As can be seen from the spectrum and spectrogram, analyzing spectrogram can be known the specific time period of useful transient information. Thus, it can be very easy to extract useful diagnostic information through the analysis of pathological in medicine.

\section{REFERENCES}

1. R. Princy, P. Thamarai, B.Karthik," Denoising Eeg Signal Using Wavelet Transform", International Journal Of Advanced Research In Computer Engineering \& Technology (Ijarcet) Volume 4 Issue 3, March 2015.

2. Zhang xizheng, Yin ling, Wang weixiong, "Wavelet Time-frequency Analysis of Electroencephalogram (EEG) Processing, "(IJACSA) International Journal of Advanced Computer Science and Applications, Vol. 1, No. 5, November 2010.

3. Justin Dauwels_ and Franc, ois Vialatte," Topics in Brain Signal Processing" Oxford University Press, 2006

4. Jigar D. Shah, M. S. Panse, "Eeg Purging Using Labview Based Wavelet Analysis," Ncci 2010 National Conference On Computational Instrumentation Csio Chandigarh, India, 19-20 March 2010.

5. Croft RJ, Barry RJ,'Removal of ocular artifact from the EEG', a review. Neurophysiologie clinique 2000; 30(1): 5-19.

6. Gasser TH, Ziegler P, Gattaz WF," The deleterious effects of ocular artefacts on the quantitative EEG, and a remedy", Eur Arch Psychiatry Neurosci 1992; 241: 352-6.

7. Romero S, Mananas MA, Barbanoj MJ," A comparative study of automatic techniques for ocular artifact reduction in spontaneous EEG signals based on clinical target variables",a simulation case. Computers in biology and medicine 2008; 38(3): 348-60.

8. Tong L, Liu RW, Soon WC, Huang YF,'Indeterminacy and identifiability of blind identification", IEEE Trans Circuits Syst 1991; 5: 499-509.
9. Belouchrani A, Abed-Meraim K, Cardoso JF, Moulines E," A blind source separation technique using second-order statistics", IEEE Trans Signal Process 1997; 45: 434-444.

10. Algimantas Juozapavi Ciusa, Gytis Bacevi`Ciusa, Dmitrijus Bugelskisa,"Eeg Analysis - Automatic Spike Detection", Nonlinear Analysis: Modelling And Control, 2011, Vol. 16, No. 4, 375-386.

11. Sander Koelstra, Mohammad Soleymani, Ashkan Yazdani,Antonnijholt" Deap: A Database For Emotion Analysis Using Physiological Signal", Ieee Transactions On Affective Computing, Vol. 3, No. 1, January-March 2012.

12. A. Lay-Ekuakille, P. Vergallo, D. Caratelli, F. Conversano, S. Casciaro, And A. Trabacca," Multispectrum Approach In Quantitative Eeg: Accuracy And Physical Effort", Ieee Sensors Journal, Vol.10, N.1, Pp.25-33, 2013.

13. G. Geetha, Dr.S.N.Geethalakshmi," Eeg DeNoising Using Sure Thresholding Based On Wavelet Transforms" International Journal Of Computer Applications Page No.0975 8887, Volume-24,No.6,June2011.

14. C.Vigneshwari, V.Vimala, S.Vaira Vignesh, G.Sumithra," Analysis of Finger Movements Using EEG Signal", International Journal of Emerging Technology and Advanced Engineering, Volume 3, Issue 1, January 2013. 